\title{
Integración de las Tecnologías de la Información y Comunicación en un Modelo de Enseñanza Flexible. El caso del Centro Universitario de los Valles de Guadalajara, México
}

\author{
César Calderón-Mayorga ${ }^{(1)}$, Julio Ruiz-Palmero(2) y José Sánchez-Rodríguez ${ }^{(3)}$ \\ (1) Universidad de Guadalajara, Centro Universitario de los Valles, Carretera Guadalajara-Ameca Km. 45.5, \\ 46600 Jalisco, México (email: cesarcalderon_mayorga@hotmail.com) \\ (2) Universidad de Málaga, Facultad de Ciencias de la Educación, Campus de Teatinos s/n - CP 29071, \\ Málaga, España (email: julio@uma.es) \\ (3) Universidad de Málaga, Facultad de Ciencias de la Educación, Campus de Teatinos s/n - CP 29071, \\ Málaga, España (email: josesanchez@uma.es)
}

Recibido Mar. 4, 2016; Aceptado Abr. 20, 2016; Versión final Abr. 27, 2016, Publicado Oct. 2016

\begin{abstract}
Resumen
El objetivo del estudio es analizar el uso que hacen los docentes de la tecnología que el Centro Universitario de los Valles de Guadalajara en México pone a su disposición para llevar a cabo el modelo de enseñanza flexible que desarrollan. Se explora y discute especialmente las razones por las que las utilizan, así como las dificultades encontradas en su uso. El estudio realizado se enmarca dentro de una metodología descriptiva y mixta, en la que se aplicó un cuestionario y se realizaron entrevistas, comparándose los resultados obtenidos para las diferentes licenciaturas. Con esto se logra identificar los principales usos que realizan, constatando de esta manera que, a pesar de su importancia en la modalidad educativa de la institución, se encuentra subutilizada. Con estos resultados, el estudio se puede extender a las demás licenciaturas para establecer acciones que contribuyan a mejorar su utilización por parte de los docentes en beneficio de los estudiantes.
\end{abstract}

Palabras clave: aprendizaje mixto; plataforma; aprendizaje flexible; tecnologías de la información y comunicación; TIC

\section{Integration of Information and Communication Technologies in a Flexible Teaching Model. The case of the University Center of the Valleys of Guadalajara, Mexico}

\begin{abstract}
The aim of this study is to analyze the use of technology that professors of the University Center of the Valleys of Guadalajara in Mexico are employing to comply with the flexible teaching model of the Center. The reason for their use and the difficulties encountered in their implementation are specially discussed. A descriptive and mixed type methodology is used including questionnaires and interviews, comparing results for the different majors. In this way the main uses of the flexible teaching model were identified, finding that, despite the importance of this educational model for the institution, technology is not properly used. With the results of the study should be extended to other degrees in order to establish actions to help improving their use by teachers for the benefit of students.
\end{abstract}

Keywords: blended learning; platform; flexible teaching; information and communication technology; ICT 


\section{INTRODUCCIÓN}

La Universidad de Guadalajara es la principal institución de Educación Superior del Estado de Jalisco, México, así como del occidente del país y la segunda en importancia a nivel nacional. En lo correspondiente al nivel Superior que atiende, está integrada por Centros Universitarios localizados en la Zona Metropolitana de Guadalajara así como por Centros Universitarios Regionales localizados en las principales regiones del Estado de Jalisco. El Centro Universitario de los Valles (Cuvalles) es un Centro Regional localizado en Ameca, en la Región de los Valles del Estado de Jalisco, que inició actividades en el año 2000 con cuatro Licenciaturas, llegando a ofertar hoy en día once. Desde el inicio de sus actividades Cuvalles impulsó una modalidad educativa en las que el alumnado asiste solo dos o tres días por semana a sesiones presenciales, y el resto de las actividades se apoyan en actividad en línea.

Tanto en Cuvalles como en muchas universidades el desarrollo de Internet y de las Tecnologías de la Información y la Comunicación, en adelante TIC, han contribuido a incorporar el trabajo en línea en sus cursos. Con relación a la primera, Cabero (2010) señala que ha hecho que los ordenadores pasen de ser instrumentos de presentación y tratamiento de la información, a ser herramientas de comunicación e interacción social. En el mismo sentido Tello, Miguel y López (2012) consideran que las potencialidades que Internet ofrece al ámbito educativo son inmensas; sin embargo, señalan que es importante saber cómo utilizarlo adecuadamente para aprovechar sus posibilidades y mejorar los procesos de enseñanza y aprendizaje, no centrándose únicamente en su implementación. Por su parte, Cabero (2010) afirma que las TIC son medios significativos para el aprendizaje, entornos de innovación escolar, así como para la comunicación e interacción social. Afirma, igualmente, que han ofrecido un gran cúmulo de oportunidades para su aplicación en el ámbito educativo.

Para el Banco Interamericano de Desarrollo las TIC pueden apoyar la mejora de la calidad educativa, obligando al cambio de las prácticas docentes así como de los sistemas escolares, permitiendo pensar en una educación masiva pero a la vez personalizada, facilitando el trabajo de los docentes con relación a los aprendizajes de los estudiantes, necesitando y logrando con ello que el rol de profesor sea diferente (Cabrol y Severin, 2010). Severin (2010) sostiene que el empleo de las TIC genera la expectativa de que permitirán modificar las prácticas de enseñanza por parte de los docentes así como de aprendizaje por parte del alumnado, es decir los roles que cada uno de ellos ha desempeñado tradicionalmente. Sin embargo, esto es lo que idealmente se desearía que sucediera, ya que en la práctica existe resistencia a modificar dichos roles, en muchas ocasiones se incorpora la tecnología pero sin modificar el desarrollo habitual de los cursos, no modificándose la práctica educativa, al menos como podría suponerse. Por otra parte, Valenzuela-Zambrano y Pérez-Villalobos (2013) señalan que el impulso que ha dado Internet ha hecho posible la enseñanza virtual, siendo parte fundamental para ello las plataformas educativas, las cuales permiten crear un entorno virtual de aprendizaje con facilidad.

En el caso de Cuvalles el uso de las plataformas virtuales es fundamental por su modalidad educativa en la que se combina la presencialidad con el trabajo en línea; también lo es para sus docentes y, en particular para su alumnado. En el caso de estos últimos muchos viven en lugares distantes del Centro Universitario, lo que implica traslados que suponen grandes distancias y tiempo, por ello el apoyarse en esta plataforma ha contribuido al desarrollo de los cursos así como de los aprendizajes vinculados a ellos, sin depender de la necesidad de asistir todos los días como sucede en la mayoría de modalidades presenciales.

\section{EL BLENDED LEARNING EN LOS PROCESOS DE APRENDIZAJE}

El trabajo en línea de esta modalidad educativa de Cuvalles permite combinar presencialidad con el trabajo en línea, hablamos por tanto de blended learning. Sobre esta modalidad, Vaughan, Cleveland-Innes y Garrison (2013) afirman que combina actividades cara a cara con el aprendizaje en línea, fusionando lo mejor de ambas. Los mismos autores señalan que la principal función del blended learning es extender el pensamiento y el discurso más allá del tiempo y el espacio, algo fundamental en Cuvalles dado el lugar de origen de muchos de los estudiantes, permitiéndoles continuar con el desarrollo de sus estudios y sus aprendizajes desde sus casas.

Por otro lado, Morán (2012) mantiene que el blended learning comienza a aparecer desde la enseñanza presencial, intentando combinar esta última con la tecnología no presencial para poder seleccionar medios adecuados a cada necesidad educativa. Las instituciones de educación superior cada vez más utilizan un amplio rango de enfoques de blended learning en sus cursos intentando enriquecer los entornos de aprendizaje (Demirer y Sahin, 2013), modificando los roles tradicionales de docentes y estudiantes. El alumnado se convierte en protagonista de su proceso educativo, en tanto que el docente se centra en diseñar estrategias de aprendizaje (Valenzuela-Zambrano y Pérez-Villalobos, 2013; Cardona y Sánchez, 2010). Parece evidente, por tanto, que los roles tradicionales de docentes y estudiantes se deberían modificar al apoyarse en la tecnología, pues en la actualidad los estudiantes pueden acceder a materiales 
de los cursos en cualquier momento, permitiéndoles personalizar su aprendizaje, adaptarse a sus horarios singulares y necesidades (Karam, 2014). En el caso de Cuvalles es muy relevante, ya que de lo contrario estaría en riesgo la consecución de los objetivos de los diferentes estudios, así como el logro del perfil profesional que se pretende lograr en los egresados de las diferentes licenciaturas.

En relación con la enseñanza en línea hay que tener en cuenta que no se trata de publicar materiales tradicionales del aula en una plataforma de aprendizaje (Morris, 2010), por lo que se debe ir más allá generando estrategias y procesos que aprovechen sus ventajas e innovaciones que contribuyan al logro de los aprendizajes de los estudiantes. El aprendizaje en línea permite alternativas flexibles al modelo tradicional, como permitir al alumnado aprender sin restricciones de espacio o tiempo, teniendo en ello un rol esencial las plataformas de aprendizaje. Coincidimos con Días y Diniz (2014) en que las plataformas de aprendizaje han sido estructuradas para administrar actividades de enseñanza y aprendizaje en línea, permiten la comunicación, la interacción y el compartir información; sin embargo, en muchos casos solo se utilizan para distribuir información, en lugar de aprovechar todo su potencial en beneficio del aprendizaje del estudiante.

Dentro de las plataformas de aprendizaje Moodle es la que tiene mayor implantación entre ellas (Williams van Rooij, 2012). Se trata de una plataforma de software libre, lo que representa la ventaja de no requerir el pago de una licencia, permitir que el código sea modificado, además de proporcionar a los docentes las mejores herramientas para la administración y promoción del aprendizaje (Marín y Maldonado, 2011). Entre sus ventajas Bošković, Gajić y Tomić (2014) destacan la comunicación del alumnado con los docentes y el acceso a materiales y actividades del curso. Al utilizar Moodle como un aula virtual los docentes pueden gestionar contenidos, favorecer la comunicación, solicitar y recibir tareas, evaluar las actividades (Fariña, González y Area, 2013), etc. Además de ello mantener contacto permanente con los estudiantes y favorecer un proceso de formación continuo que no solo se desarrolle en los espacios físicos de las universidades, contribuyendo con ello al logro de los objetivos de aprendizaje que se busca logren los estudiantes.

Con relación a las plataformas de aprendizaje se han realizado diversos estudios sobre ellas desde diferentes perspectivas: costo, funcionalidad, facilidad de uso, adquisición e implementación (Muñoz-Cano et al., 2012; Williams van Rooij, 2012); en otros casos, para conocer las habilidades tecnológicas de los estudiantes que las utilizan (Maldonado y Vega, 2015); así como evaluar las competencias tecnológicas de los profesores que utilizan esta plataforma (Mirabal, Gómez y González, 2015); o bien los usos por parte de los estudiantes como apoyo en su proceso de enseñanza aprendizaje (Lamas, Massié y Quero, 2010; Marín y Maldonado, 2010); incluso también se han llevado a cabo estudios de la plataforma como herramienta para la investigación (Moreno, Pérez y del Olmo, 2013).

Dada la importancia que tiene para Cuvalles por su modalidad educativa, se hace necesario evaluar la percepciones que tienen de ella los docentes de varias licenciaturas, así como la importancia que tiene desde su perspectiva su empleo, ya que al ser ellos actores de gran importancia en su utilización, de como ellos la utilicen depende en buena medida el éxito de la modalidad educativa de Cuvalles. También se busca identificar similitudes y en su caso diferencias en la forma en que es utilizada la plataforma entre los profesores de las cuatro licenciaturas en cuestión.

\section{METODOLOGÍA}

Se presenta el diseño y los objetivos del estudio, se describe la población y se detallan los iinstrumentos aplicados.

\section{Diseño y objetivos}

Los objetivos en esta investigación fueron conocer y comprender el uso que hacen los docentes de las herramientas de Moodle, las razones por las que las utilizan, así como las dificultades encontradas en su empleo en las licenciaturas en Administración, Contaduría Pública, Ingeniería en Electrónica y Computación e Ingeniería Mecatrónica, además de identificar las similitudes y diferencias en el uso que se hace de ellas entre los profesores de estas licenciaturas.

Se utilizó un enfoque mixto que, de acuerdo con Creswell, Plano, Gutmann y Hanson (2003), contempla la recogida o análisis de datos cuantitativos y/o cualitativos, para lo cual los datos se recogen secuencialmente o concurrentemente, logrando la integración de datos en una o más etapas del proceso de investigación. En el caso de las metodologías mixtas, Creswell (2009) contempla los diseños secuenciales y los diseños concurrentes; en este caso se utilizó un diseño secuencial explicativo, el cual en la primer etapa se caracteriza por la recogida y análisis de datos cuantitativos y en la segunda se realiza la recogida y análisis de datos cualitativos, basados en los resultados cuantitativos iniciales. En este tipo de diseños Creswell et al. (2003) afirman que los métodos se integran en la fase de interpretación del estudio. 
La investigación realizada se desarrolló considerando las siguientes fases: 1) Elaboración del cuestionario, que fue completado en línea a través de la plataforma Limesurvey por todos los docentes de las cuatro licenciaturas; 2) Realización de análisis estadístico de los resultados; 3) Entrevistas a todos los docentes de las cuatro licenciaturas y transcripción para su análisis; y 4) Contraste de los resultados de cuestionarios y entrevistas.

\section{Población}

El estudio se desarrolló considerando la totalidad de docentes de las licenciaturas en Administración, Contaduría Pública, Ingeniería en Electrónica y Computación e Ingeniería Mecatrónica de Cuvalles durante los años 2012 y 2013, por lo que no requirió de ninguna técnica muestral. En la tabla 1 se puede observar la distribución del profesorado por licenciatura; el 24,51 \% pertenecía a los estudios de Contaduría, el 31,37\% a los de Administración, el 21,57\% a los de Mecatrónica y el 22,55\% a Electrónica y Computación.

Tabla 1: Distribución de profesores por licenciatura $(\mathrm{N}=102)$

\begin{tabular}{|l|c|c|}
\hline & $\mathrm{N}$ & $(\%)$ \\
\hline Contaduría & 25 & 24,51 \\
\hline Administración & 32 & 31,37 \\
\hline Mecatrónica & 22 & 21,57 \\
\hline Electrónica y Computación & 23 & 22,55 \\
\hline
\end{tabular}

\section{Instrumentos}

Se utilizó un cuestionario "ad hoc" integrado por 18 preguntas cerradas que cumplimentaron 102 profesores. En el análisis de datos se empleó el paquete estadístico SPSS 21.0 realizándose diferentes análisis descriptivos univariados y bivariados. El cuestionario tenía una fiabilidad de 0,857 (Alfa de Cronbach). Una vez recogida toda la información procedimos al análisis de los mismos siguiendo las pautas de Lukas y Santiago (2009): Etapa 1: reducción de la información, selección e identificación de la misma, establecimiento de un sistema de categorías y codificación; Etapa 2: organización de la información; y Etapa 3: análisis e interpretación de resultados.

En lo referente a las entrevistas, las mismas fueron estructuradas y constaban de siete preguntas abiertas, que se grabaron en su totalidad al contar con la autorización de los docentes; en las respuestas se buscó identificar aquellos temas, ideas o aspectos relevantes, asignándose códigos a cada uno de ellos. Con la información se fueron agrupando por categorías. Por la propia naturaleza de las entrevistas, en algunos casos ya se contaba con categorías iniciales; sin embargo, otras fueron surgiendo con el análisis de las respuestas obtenidas. Al concluir el análisis realizado a cada una de las respuestas de las preguntas, se analizó la información también en su conjunto, a la vez se contrastaron las respuestas obtenidas en las cuatro licenciaturas. Además, se realizó una interpretación de todos los resultados obtenidos buscando: lograr una visión amplia e integral de las herramientas de Moodle que utilizan los docentes y comprender la experiencia que viven día a día los docentes al utilizarlas. Para la sistematización de los datos obtenidos en las 102 entrevistas, se contó con el apoyo del programa Altas Ti.

Tabla 2: Frecuencia de uso de la herramienta foro (\%)

\begin{tabular}{|l|c|c|c|c|c|}
\hline & Muy frecuent. & Frecuentemente & Ocasionalmente & A veces & Nunca \\
\hline Administración & 53,13 & 28,13 & 9,38 & 9,38 & 0,00 \\
\hline Contaduría & 48,00 & 36,00 & 12,00 & 4,00 & 0,00 \\
\hline $\begin{array}{l}\text { Electrónica y } \\
\text { Computación }\end{array}$ & 31,82 & 36,36 & 18,18 & 9,09 & 4,55 \\
\hline Mecatrónica & 33,33 & 28,57 & 19,05 & 9,52 & 9,52 \\
\hline
\end{tabular}

\section{RESULTADOS}

Con relación a la frecuencia con la que los docentes utilizan las herramientas de Moodle, es posible identificar que los foros son lo más utilizado, siendo la opción muy frecuentemente la predominante en tres de las licenciaturas (tabla 2): Administración con un 53,13\%, Contaduría Pública con un $48 \%$ e Ingeniería Mecatrónica con un 33,33\%. Solo en Ingeniería en Electrónica y Computación la opción frecuentemente fue la predominante con un $36,36 \%$. Sin embargo, la herramienta más utilizada por los docentes es la tarea, siendo la opción muy frecuentemente la predominante en las cuatro licenciaturas (tabla 3). 
Tabla 3: Frecuencia de uso de la herramienta tareas (\%)

\begin{tabular}{|l|c|c|c|c|c|}
\hline & Muy frecuent. & Frecuentemente & Ocasionalmente & A veces & Nunca \\
\hline Administración & 75,00 & 18,75 & 3,13 & 0,00 & 3,13 \\
\hline Contaduría & 84,0 & 16,00 & 0,00 & 0,00 & 0,00 \\
\hline $\begin{array}{l}\text { Electrónica y } \\
\text { Computación }\end{array}$ & 72,73 & 22,73 & 0,00 & 0,00 & 4,55 \\
\hline Mecatrónica & 80,95 & 19,05 & 0,00 & 0,00 & 0,00 \\
\hline
\end{tabular}

Acerca de las herramientas que los docentes consideran de mayor importancia, el foro es una de ellas (tabla 4), ya que es la opción predominante en dos de las licenciaturas: Administración con un $50 \%$ y en Contaduría Pública con un $52 \%$ como muy importante, mientras que en las otras dos la opción importante fue la predominante (Ingeniería en Electrónica y Computación: 45,45\% e Ingeniería Mecatrónica: 42,86\%). No obstante, la que consideran tiene mayor importancia es la tarea, siendo la opción muy importante la predominante en las cuatro licenciaturas (tabla 5).

Con respecto a los principales usos que los docentes hacen de las herramientas de Moodle la opción predominante en las cuatro licenciaturas es facilitar materiales de estudio (tabla 6) y recibir tareas, con los mismos resultados a excepción del caso de Ingeniería Mecatrónica con 95,24\% y 90,48\% respectivamente. Un uso importante en lo representa igualmente dar avisos.

Tabla 4: Importancia del uso de la herramienta Foro (\%)

\begin{tabular}{|l|c|c|c|c|c|}
\hline & $\begin{array}{c}\text { Muy } \\
\text { Importante }\end{array}$ & Importante & $\begin{array}{c}\text { Moderadamente } \\
\text { Importante }\end{array}$ & $\begin{array}{c}\text { Poco } \\
\text { Importante }\end{array}$ & Sin Importancia \\
\hline Administración & 50,00 & 43,75 & 6,25 & 0,00 & 0,00 \\
\hline Contaduría & 52,0 & 40,00 & 8,00 & 0,00 & 0,00 \\
\hline $\begin{array}{l}\text { Electrónica y } \\
\text { Computación }\end{array}$ & 36,36 & 45,45 & 18,18 & 0,00 & 0,00 \\
\hline Mecatrónica & 38,10 & 42,86 & 14,29 & 0,00 & 4,76 \\
\hline
\end{tabular}

Tabla 5: Importancia del uso de la herramienta Tareas (\%)

\begin{tabular}{|l|c|c|c|c|c|}
\hline & $\begin{array}{c}\text { Muy } \\
\text { Importante }\end{array}$ & Importante & $\begin{array}{c}\text { Moderadamente } \\
\text { Importante }\end{array}$ & $\begin{array}{c}\text { Poco } \\
\text { Importante }\end{array}$ & $\begin{array}{c}\text { Sin } \\
\text { Importancia }\end{array}$ \\
\hline Administración & 81,25 & 18,75 & 0,00 & 0,00 & 0,00 \\
\hline Contaduría & 76,0 & 24,00 & 0,00 & 0,00 & 0,00 \\
\hline $\begin{array}{l}\text { Electrónica y } \\
\text { Computación }\end{array}$ & 81,82 & 13,64 & 4,55 & 0,00 & 0,00 \\
\hline Mecatrónica & 80,95 & 19,05 & 0,00 & 0,00 & 0,00 \\
\hline
\end{tabular}

Tabla 6: Principales usos de las herramientas de Moodle (\%)

\begin{tabular}{|l|c|c|c|c|c|c|}
\hline & Avisos & $\begin{array}{c}\text { Analizar } \\
\text { temas }\end{array}$ & $\begin{array}{c}\text { Recibir } \\
\text { tareas }\end{array}$ & $\begin{array}{c}\text { Aplicar } \\
\text { Exámenes }\end{array}$ & $\begin{array}{c}\text { Facilitar } \\
\text { Materiales }\end{array}$ & Asesoría \\
\hline Administración & 93,75 & 68,75 & 96,88 & 50,00 & 96,88 & 62,54 \\
\hline Contaduría & 88,00 & 76,00 & 100 & 64,00 & 100 & 72,00 \\
\hline $\begin{array}{l}\text { Electrónica y } \\
\text { computación }\end{array}$ & 90,91 & 54,55 & 95,45 & 59,09 & 95,45 & 50,00 \\
\hline Mecatrónica & 90,48 & 42,86 & 90,48 & 52,38 & 95,24 & 47,62 \\
\hline
\end{tabular}

En lo correspondiente a los beneficios que los docentes perciben al utilizar las herramientas de Moodle (tabla 7), en Administración predomina la opción ayudar a mantener la comunicación con los estudiantes $(96,88 \%)$, en Contaduría permitir facilitar materiales de estudio junto con apoyar las sesiones presenciales (ambas con un 92\%) y en Ingeniería en Electrónica y Computación e Ingeniería Mecatrónica permitir facilitar materiales de estudio (con un $90,91 \%$ y un $95.24 \%$ respectivamente). Por tanto, en tres de cuatro licenciaturas predomina el permitir facilitar materiales de estudio como el principal beneficio de utilizar las herramientas de Moodle. 
Tabla 7: Principales beneficios de las herramientas de Moodle (\%)

\begin{tabular}{|l|c|c|c|c|c|c|}
\hline & $\begin{array}{c}\text { Facilitar } \\
\text { materiales } \\
\text { de estudio }\end{array}$ & $\begin{array}{c}\text { Ayudar a } \\
\text { mantener } \\
\text { comunicación }\end{array}$ & $\begin{array}{c}\text { Facilitar } \\
\text { evaluación } \\
\text { de } \\
\text { actividades }\end{array}$ & $\begin{array}{c}\text { Continuar } \\
\text { con } \\
\text { desarrollo } \\
\text { del curso }\end{array}$ & $\begin{array}{c}\text { Apoyar } \\
\text { sesiones } \\
\text { presenciales }\end{array}$ & $\begin{array}{c}\text { No hay } \\
\text { beneficio }\end{array}$ \\
\hline Administración & 93,75 & 96,88 & 71,88 & 78,13 & 84,38 & 0,00 \\
\hline Contaduría & 92,00 & 88,00 & 80,00 & 84,00 & 92,00 & 0,00 \\
\hline $\begin{array}{l}\text { Electrónica y } \\
\text { Computación }\end{array}$ & 90,91 & 77,27 & 63,64 & 59,09 & 81,82 & 0,00 \\
\hline Mecatrónica & 95,24 & 66,67 & 52,38 & 52,38 & 76,19 & 0,00 \\
\hline
\end{tabular}

Con relación a los objetivos que buscan los docentes al utilizar las herramientas de Moodle, en todos los casos la opción predominante es fomentar el autoaprendizaje (tabla 8). Cabe señalar que en el caso de Ingeniería en Electrónica y Computación la opción proporcionar recursos y materiales obtuvo también un $81,82 \%$.

Al preguntar a los profesores acerca de las principales dificultades que identifican al utilizar las herramientas de Moodle (tabla 9), la opción predominante fue la conexión lenta a Internet. Cabe señalar que en el caso de Contaduría Pública hay dos opciones más con el mismo porcentaje: la dificultad en la conexión a la página de Moodle y la falta de tiempo para utilizar las herramientas.

También se analizaron las posibles correlaciones existentes entre las distintas variables analizadas en las diferentes licenciaturas; en el caso de la licenciatura en Administración destaca la relación $(0,636)$ entre principales beneficios y objetivos buscados (tabla 10). Por otra parte, se identificó una correlación $(0,652)$ entre objetivos buscados y aportaciones a los alumnos (tabla 11).

Tabla 8: Objetivos buscados al utilizar las herramientas de Moodle (\%)

\begin{tabular}{|l|r|r|r|r|r|r|}
\hline & $\begin{array}{c}\text { Continuar } \\
\text { con el } \\
\text { desarrollo } \\
\text { del curso }\end{array}$ & $\begin{array}{c}\text { Fomentar el } \\
\text { autoaprendizaje }\end{array}$ & $\begin{array}{c}\text { Mantener } \\
\text { comunicación } \\
\text { constante }\end{array}$ & $\begin{array}{c}\text { Fomentar } \\
\text { aprendizaje } \\
\text { con } \\
\text { tecnología }\end{array}$ & $\begin{array}{c}\text { Proporcionar } \\
\text { recursos y } \\
\text { materiales }\end{array}$ & $\begin{array}{c}\text { Informar } \\
\text { avances }\end{array}$ \\
\hline Administración & 78,13 & 96,88 & 56,25 & 59,38 & 87,50 & 31,25 \\
\hline Contaduría & 84,00 & 100 & 68,00 & 72,00 & 84,00 & 44,00 \\
\hline $\begin{array}{l}\text { Electrónica y } \\
\text { Computación }\end{array}$ & 54,55 & 81,82 & 50,00 & 54,55 & 81,82 & 31,82 \\
\hline Mecatrónica & 66,67 & 71,43 & 42,86 & 61,90 & 76,19 & 28,57 \\
\hline
\end{tabular}

Tabla 9: Principales dificultades al utilizar las herramientas de Moodle (\%)

\begin{tabular}{|l|c|c|c|c|c|c|}
\hline & $\begin{array}{c}\text { Fallas en el } \\
\text { equipo de } \\
\text { cómputo }\end{array}$ & $\begin{array}{c}\text { Dificultad en } \\
\text { la conexión con } \\
\text { la página } \\
\text { de Moodle }\end{array}$ & $\begin{array}{c}\text { Conexión } \\
\text { lenta a } \\
\text { internet }\end{array}$ & $\begin{array}{c}\text { Falta de } \\
\text { capacitación }\end{array}$ & $\begin{array}{c}\text { Falta de tiempo } \\
\text { para utilizar las } \\
\text { herramientas }\end{array}$ & $\begin{array}{c}\text { Falta de } \\
\text { herramientas } \\
\text { adecuadas }\end{array}$ \\
\hline Administración & 78,13 & 96,88 & 56,25 & 59,38 & 87,50 & 31,25 \\
\hline Contaduría & 84,00 & 100 & 68,00 & 72,00 & 84,00 & 44,00 \\
\hline $\begin{array}{l}\text { Electrónica y } \\
\text { Computación }\end{array}$ & 54,55 & 81,82 & 50,00 & 54,55 & 81,82 & 31,82 \\
\hline Mecatrónica & 66,67 & 71,43 & 42,86 & 61,90 & 76,19 & 28,57 \\
\hline
\end{tabular}

Tabla 10: Correlación entre principales beneficios y objetivos buscados en Administración

\begin{tabular}{|l|l|r|r|}
\hline & & Principales beneficios & Objetivos buscados \\
\hline Principales beneficios & Correlación de Pearson & 1 &, 636 \\
\hline & Sig. (bilateral) & &, 000 \\
\hline & $\mathrm{N}$ & 32 & 32 \\
\hline Objetivos buscados & Correlación de Pearson &, 636 & 1 \\
\hline & Sig. (bilateral) &, 000 & 32 \\
\hline & $\mathrm{N}$ & 32 & \\
\hline
\end{tabular}


Tabla 11: Correlación entre objetivos buscados y aportaciones a alumnos en Administración.

\begin{tabular}{|l|l|r|r|}
\hline & & $\begin{array}{r}\text { Objetivos } \\
\text { buscados }\end{array}$ & $\begin{array}{r}\text { Aportaciones a } \\
\text { alumnos }\end{array}$ \\
\hline $\begin{array}{l}\text { Objetivos } \\
\text { buscados }\end{array}$ & $\begin{array}{l}\text { Correlación de } \\
\text { Pearson }\end{array}$ & 1 &, 636 \\
\hline & Sig. (bilateral) & 32 &, 000 \\
\hline & $\mathrm{N}$ &, 636 & 32 \\
\hline $\begin{array}{l}\text { Aportaciones a } \\
\text { alumnos }\end{array}$ & $\begin{array}{l}\text { Correlación de } \\
\text { Pearson }\end{array}$ &, 000 & 1 \\
\hline & Sig. (bilateral) & 32 & 32 \\
\hline & $\mathrm{N}$ & & \\
\hline
\end{tabular}

En el caso de Contaduría Pública se encontró correlación $(0,630)$ entre objetivos buscados y aportaciones al alumnado (tabla 12); de igual forma, se encontró correlación $(0,535)$ entre principales beneficios y objetivos buscados (tabla 13). En el caso de Ingeniería en Electrónica y Computación se encontró correlación $(0,824)$ entre principales usos y principales beneficios (tabla 14) y entre principales beneficios y objetivos buscados $(0,723)$ (tabla 15). En el caso de Ingeniería Mecatrónica se encontró correlación $(0,802)$ entre principales usos y principales beneficios (tabla 16) y entre principales beneficios y objetivos buscados $(0,803$ ) (tabla 17).

Tabla 12: Correlación entre objetivos buscados y aportaciones a alumnos en Contaduría Pública

\begin{tabular}{|l|l|r|r|}
\hline & & Objetivos buscados & Aportaciones a alumnos \\
\hline Objetivos buscados & Correlación de Pearson & 1 &, 630 \\
\hline & Sig. (bilateral) & &, 000 \\
\hline & N & 25 & 25 \\
\hline Aportaciones a alumnos & Correlación de Pearson &, 630 & 1 \\
\hline & Sig. (bilateral) &, 000 & 25 \\
\hline & N & 25 & \\
\hline
\end{tabular}

Tabla 13: Correlación entre principales beneficios y objetivos buscados en Contaduría Pública

\begin{tabular}{|l|l|r|r|}
\hline & & Objetivos buscados & Aportaciones a alumnos \\
\hline Objetivos buscados & Correlación de Pearson & 1 &, 535 \\
\hline & Sig. (bilateral) & &, 000 \\
\hline & $\mathrm{N}$ & 25 & 25 \\
\hline Aportaciones a alumnos & Correlación de Pearson &, 535 & 1 \\
\hline & Sig. (bilateral) &, 000 & 25 \\
\hline & $\mathrm{N}$ & 25 & \\
\hline
\end{tabular}

Tabla 14: Correlación entre principales usos y principales beneficios en Ingeniería en Electrónica y Computación

\begin{tabular}{llrr}
\hline & & Principales usos & Principales beneficios \\
\hline Principales usos & Correlación de Pearson & 1 &, 824 \\
& Sig. (bilateral) & &, 000 \\
& $\mathrm{~N}$ & 22 & 22 \\
\hline Principales beneficios & Correlación de Pearson &, 824 & 1 \\
& Sig. (bilateral) &, 000 & \\
& $\mathrm{~N}$ & 22 & 22
\end{tabular}

Tabla 15: Correlación entre principales beneficios y objetivos buscados en Ingeniería en Electrónica y Computación

\begin{tabular}{llrr}
\hline & & Principales beneficios & Objetivos buscados \\
\hline Principales beneficios & Correlación de Pearson & 1 &, 723 \\
& Sig. (bilateral) & &, 000 \\
& $\mathrm{~N}$ & 22 & 22 \\
\hline Objetivos buscados & Correlación de Pearson &, 723 & 1 \\
& Sig. (bilateral) &, 000 & \\
& $\mathrm{~N}$ & 22 & 22 \\
\hline
\end{tabular}


Tabla 16: Correlación entre principales usos y principales beneficios en Ingeniería Mecatrónica

\begin{tabular}{|l|l|r|r|}
\hline & & Principales usos & Principales beneficios \\
\hline Principales usos & Correlación de Pearson & 1 &, 802 \\
\hline & Sig. (bilateral) & &, 000 \\
\hline & N & 21 & 21 \\
\hline Principales beneficios & Correlación de Pearson &, 802 & 1 \\
\hline & Sig. (bilateral) &, 000 & 21 \\
\hline & N & 21 & \\
\hline
\end{tabular}

Tabla 17: Correlación entre principales beneficios y objetivos buscados en Ingeniería Mecatrónica.

\begin{tabular}{|l|l|r|r|}
\hline & & Principales beneficios & Objetivos buscados \\
\hline Principales beneficios & Correlación de Pearson & 1 & .803 \\
\hline & Sig. (bilateral) & & .000 \\
\hline & $\mathrm{N}$ & 21 & 21 \\
\hline Objetivos buscados & Correlación de Pearson & .803 & 1 \\
\hline & Sig. (bilateral) & .000 & 21 \\
\hline & $\mathrm{N}$ & 21 & \\
\hline
\end{tabular}

De las entrevistas realizadas destacamos que en las cuatro licenciaturas los docentes consideran importante utilizar la plataforma Moodle y sus herramientas por la modalidad educativa de Cuvalles, siendo las más utilizadas el foro, las tareas y el cuestionario. Coinciden en las razones por las que las utilizan al señalar que las emplean porque son las que saben utilizar, pues les facilita la recepción de tareas, la comunicación con los estudiantes, así como por la necesidad de utilizarlas por el modelo académico de Cuvalles. También expresaron que existe buena respuesta del alumnado al utilizarlas. Del mismo modo, consideran necesario que se les brinde capacitación para utilizar las demás herramientas.

Se puede decir que existe una buena percepción por parte de los docentes en la utilización de las herramientas de Moodle, a la vez que reconocen la importancia que tiene para el modelo académico de la institución; sin embargo, existen docentes que indicaron no utilizarlas en absoluto en sus cursos, argumentando estar esperando tener las condiciones personales necesarias para hacerlo. En el caso particular de los docentes de las dos ingenierías expresaron la dificultad que representa para ellos el utilizar las herramientas en los cursos de Matemáticas.

\section{DISCUSIÓN}

Al contrastar las respuestas obtenidas del cuestionario con las de las entrevistas observamos coincidencias en lo concerniente a las herramientas que son más utilizadas: tarea, foro y cuestionario. Del mismo modo se observa que las herramientas que utilizan con más frecuencia son las que a su vez consideran más importantes, siendo estas las mencionadas previamente.

De igual forma, los principales usos que hacen de las herramientas de Moodle, es para recibir tareas y facilitar materiales y avisos, usos que corresponden a las herramientas que se utilizan con mayor frecuencia y que, a su vez, consideran de mayor importancia. En lo correspondiente a los beneficios de utilizar estas herramientas que identifican los docentes destacan: facilitar materiales de estudio, ayudar a mantener la comunicación con el alumnado, así como el apoyar las sesiones presenciales, coincidiendo con lo aportado por Lamas, Massié y Quero (2010). Por otra parte con relación a los objetivos que los docentes buscan al utilizar estas herramientas, señalan que son fomentar el autoaprendizaje así como proporcionar recursos y materiales. En lo concerniente a las principales dificultades identificadas al utilizar las herramientas de Moodle, resaltan los fallos de conexión a la web de Moodle, falta de tiempo para utilizarlas y fallos en los equipos que dan acceso.

De las entrevistas podemos rescatar la importancia que tiene para los docentes, independientemente de la licenciatura en la que participan, el empleo de la plataforma Moodle y sus herramientas, de acuerdo con estudios previos (Padilla et al., 2015). También es destacable que las herramientas que más utilizan sean foro, tareas y cuestionario, siendo tareas la más utilizada. Cabe mencionar que afirmaron emplear estas herramientas porque son las que saben utilizar, reconociendo la necesidad de capacitación, en la línea de lo aportado por Fariña, González y Area (2013). Del mismo modo, llama la atención que unos pocos profesores hayan afirmado no utilizar la plataforma y por ende las herramientas, situación que debería atenderse dada la importancia que tiene para la modalidad educativa de Cuvalles. En general, y en particular en estos casos, existe disposición de los profesores para capacitarse, situación en la que podría apoyarse la institución para generar acciones que atendieran esta necesidad. 
El Centro Universitario de los Valles de Guadalajara (México) ha impulsado y basado su trabajo formativo desde su inicio en una modalidad educativa que combina sesiones presenciales con actividad en línea a través de la plataforma Moodle. Parte esencial para que esta modalidad educativa funcione en forma adecuada en beneficio de la formación del alumnado, son los docentes.

De los resultados del estudio podemos destacar que son muy pocas las herramientas utilizadas por los docentes, y por tanto por el alumnado, ya que estos atienden la pauta establecida por el profesor. Al utilizar pocas herramientas no se aprovecha del todo el potencial de las herramientas que se ponen a su disposición, pero lo que debe preocupar más es que no se realicen las acciones que contribuyan al logro de una formación de calidad de los estudiantes, función principal de la institución.

Llama especialmente la atención que estas herramientas se empleen por ser las únicas que saben utilizar, lo que conlleva distintas implicaciones, al asistir el alumnado únicamente dos o tres días a la semana a sesiones presenciales, en los demás días se considera que deberían estar atendiendo actividades de sus diferentes cursos, para de esta forma continuar su proceso formativo desde sus lugares de origen. Sin embargo, al observar los resultados de este estudio es cuestionable la forma en que se desarrolla la actividad en Moodle, ya que al identificar las herramientas utilizadas así como los principales usos de las mismas, el trabajo en la plataforma queda limitado a proporcionar materiales, abrir buzones de tareas y enviar avisos, acciones que no contribuyen a un proceso continuo de formación del alumnado, pudiéndose afirmar que se emplea Moodle como repositorio de información y receptor de tareas.

Debido a la importancia que tiene para Cuvalles el apoyarse en Moodle, consideramos que existe la necesidad de emprender un amplio abanico de posibilidades buscando mejorar la forma en que se utiliza por parte de los docentes.

Es recomendable la capacitación de todos los docentes sobre la plataforma y sus herramientas, las estrategias a seguir en una modalidad que combina presencialidad con actividad en línea, pone de manifiesto la necesidad de hacer un esfuerzo que conlleve promover buenas prácticas en el uso de la plataforma, generando de ese modo un proceso formativo continuo en el alumnado, de lo contrario se puede poner en riesgo la formación profesional de los mismos.

Existe la disposición de los docentes para formarse y reconocen la importancia de las herramientas de las que disponen, por lo que debe aprovecharse esta circunstancia para considerar un proceso de formación continuo de todos ellos.

Finalmente, con este estudio realizado con los docentes de cuatro de las licenciaturas de Cuvalles, Administración, Contaduría Pública, Ingeniería en Electrónica y Computación e Ingeniería Mecatrónica, se concluyen resultados que pueden servir para extender este estudio a las demás licenciaturas con el fin de establecer estrategias y generar acciones en beneficio del mejor funcionamiento de la modalidad educativa pero sobre todo en beneficio de una mejor formación profesional del alumnado, sin embargo en tanto esto sucede ya se tienen elementos para emprender acciones que atiendan las situaciones aquí identificadas.

\section{CONCLUSIONES}

Del estudio realizado y de su discusión, se puede extraer las siguientes conclusiones principales:

1.- Las herramientas TIC que Cuvalles pone a disposición del profesorado están subutilizadas y no se emplean de manera adecuada, a pesar de ser fundamentales en la modalidad educativa de la institución.

2.- Se debe trabajar de una manera profusa en la capacitación de los docentes, tanto en el uso instrumental de la tecnología disponible como en actualizar las estrategias didácticas a emplear con el alumnado.

3.- Los docentes reconocen la importancia de recibir una formación adecuada y adaptada al contexto y circunstancias de Cuvalles, por lo que consideramos debe aprovecharse dicha predisposición.

4.- Los resultados y conclusiones presentadas pueden extenderse al resto de licenciaturas y de ese modo propiciar situaciones que redunden en la mejora del modelo educativo analizado.

\section{REFERENCIAS}

Bošković, V., T. Gajić, y I. Tomić, Moodle in English Language Teaching, doi: 10.15308/SInteZa-2014-480483, Singidunum Journal Of Applied Sciences, 480-483 (2014)

Cabero, J., Los retos de la integración de las TICs en los procesos educativos. Límites y posibilidades, http://www.perspectivaeducacional.cl/index.php/peducacional/article/view/3; ISSN 0718-9729, Perspectiva educacional, 49(1), 32-61 (2010) 
Cabrol, M., y E. Severin, TICs en educación: una innovación disruptiva. Banco Interamericano de Desarrollo (2010)

Cardona, D.M. y J.M. Sánchez, Indicadores básicos para evaluar el proceso de aprendizaje en estudiantes de educación a distancia en ambientes e-learning, doi: 10.4067/S0718-50062010000600004, Form. Univ., 3(6), 15-32 (2010)

Creswell, J., Research Design. Qualitative, Quantitative, and Mixed Methods Approaches. Thousand Oaks, CA, Sage (2009)

Creswell, J.W., V. L. Plano, M.L. Gutmann y W.E. Hanson, Advanced mixed methods research designs, in Handbook of mixed methods in social and behavioral research by A. Tashakkori y C. Teddlie (Eds.), pp 209240. Thousand Oaks, CA, Sage (2003)

Demirer, V.I. y I. Sahin, Effect of blended learning environment on transfer of learning: an experimental study, doi: doi:10.1111/jcal.12009, Journal Of Computer Assisted Learning, 29(6), 518-529 (2013)

Dias, S.B. y J.A. Diniz, Towards an Enhanced Learning Management System for Blended Learning in Higher Education Incorporating Distinct Learners' Profiles, http://www.ifets.info/journals/17_1/ets_17_1.pdf, ISSN 1436-4522 Educational Technology \& Society, 17(1), 307-319 (2014)

Fariña, E., C. S. González y M. Area, ¿Qué uso hacen de las aulas virtuales los docentes universitarios?, http://www.um.es/ead/red/35, ISSN: 1578-7680, RED. Revista de Educación a Distancia, 35, (2013)

Karam, E. C., Together Face-to-Face or Alone at Your Own Pace: Comparing Traditional vs. Blended Learning Formats in Couple \& Family Relationship Coursework. Journal Of Instructional Psychology, 41(1-4), 85-93 (2014)

Lamas, M.L., A.I. Massié, y E.D. Quero, Implementación de un aula virtual bajo la modalidad mixta: el caso de química agrícola en la Universidad Nacional de Salta, doi: 10.4067/S0718-50062010000400002, Form. Univ., 3(4), 3-12 (2010)

Lukas, J. F. y K. Santiago, Evaluación Educativa. Alianza, Madrid, España (2009)

Maldonado, G. y G. Vega, Actitud de los estudiantes universitarios ante la plataforma moodle, doi: 10.12795/pixelbit.2015.i47.07, Pixel-Bit. Revista de medios y educación, 47, 105-117 (2015)

Marín, V. y G. A. Maldonado, El alumnado universitario cordobés y la plataforma virtual Moodle, http://www.sav.us.es/pixelbit/actual/9.html, ISSN: 2171-7966, Pixel-Bit. Revista de Medios de Comunicación, $38,121-128$ (2011)

Mirabal, Á., M. Gómez y L. González, Uso de la plataforma Moodle como apoyo a la docencia presencial universitaria, http://www.uco.es/ucopress/ojs/index.php/edmetic/article/view/2903/2831, ISSN: 2254-0059, Revista Edmetic, 4(1), 133-155 (2015)

Morán, L., Blended-learning: Desafío y oportunidad para la educación actúa, http://www.edutec.es/revista/index.php/edutec-e/article/view/371, ISSN: 1135-9250, Edutec: Revista electrónica de tecnología educativa, 39, 1-19 (2012)

Moreno, A., C. Pérez y E. Del Olmo, Utilización de Moodle como plataforma para la investigación educativa: aplicación a los córpora de aprendices de lenguas, doi: 10.12795/pixelbit.2013.i43.09, Pixel-Bit: Revista de medios y educación, 43, 125-138 (2013)

Morris, R. D., Web 3.0: Implications for Online Learning, https://www.learntechlib.org/p/50567/, ISSN 87563894, Techtrends: Linking Research \& Practice To Improve Learning, 55(1), $42-46$ (2010)

Muñoz-Cano, J.M., J.A. Córdova, y H. PRIEGO, Dificultades y facilidades para el desarrollo de un proceso de innovación educativa con base en las Tecnologías de la Información y Comunicación (TIC), doi: 10.4067/S0718-50062012000100002, Form. Univ., 5(1), 3-12 (2012)

Severin, E., Tecnologías de la información y la comunicación (TICs) en educación. Inter-American Development Bank (2010)

Tello, I., L. D. Miguel y M. D. López, Entornos personales de aprendizaje en el espacio europeo de educación superior, http://e-spacio.uned.es/fez/eserv.php?pid=bibliuned:revistaRied-2012-15-2- 
3270\&dsID=Documento.pdf, ISSN: 1138-2783, RIED. Revista iberoamericana de educación a distancia, 15 (2), 123-142 (2012)

Valenzuela-Zambrano, B. y M. V. Pérez-Villalobos, Aprendizaje autorregulado a través de la plataforma virtual Moodle, http://educacionyeducadores.unisabana.edu.co/index.php/eye/article/view/2000/3074, ISSN: 2027-5358, Educación y Educadores: 16(1), 66-79 (2013)

Vaughan, N. D., M. Cleveland-Innes y D. R. Garrison, Teaching in Blended Learning Environments: Creating and Sustaining Communities of Inquiry. Edmonton: AU Press (2013)

Williams van Rooij, S., Open-source learning management systems: a predictive model for higher education, doi: 10.1111/j.1365-2729.2011.00422.x, Journal Of Computer Assisted Learning, 28(2), 114-125 (2012) 
